capnia but was reduced remarkably by the hypocapnia.

(B) Increased intracranial pressure group

The increasing of the intracranial pressure was made by the epidural ballooning. Each blood flows were reduced in the increased intracranial pressure; RBF and ICBF decreased more than MBF and FBF.

At $1000 \mathrm{mmH}_{2} \mathrm{O}$ balloon pressure, the effects of the acute hemorrhage under the hypercapnia and the hypocapnia on the blood flows showed the same tendency to the results of the non-increased intracranial pressure group. But ICBF under the hypercapnia was not increased so remarkably as that of the non-increased intracranial pressure group. On the other hand, the reduction of ICBF under the hypocapnia was as almost same as that of the non-increased intracranial pressure group.

\title{
g-8. Cerebral Circulation and Intracranial Hypertension
}

\author{
Takashi Ohwada, Tetsu Kuramae, Kazuyoshi Ueno, Hiroshi Abe and \\ Kenzoh YADA \\ Department of Neurosurgery, Hokkaido University School of Medicine
}

Our previous investigations of the pathophysiology of intracranial hypertension have indicated that various secondary pathophysiological changes do not always parallel to the degree of the pressure elevation but are influenced both by degree and duration of the increased intracranial pressure. Also, we have pointed out that the secondary circulatory change due to increased intracranial pressure plays an important role in further elevation of intracranial pressure. The present experimental study was made in an attempt to clarify the circulatory changes under increased intracranial pressure with special regard to time consequence. Also, it was our intension to find out which part of the cerebral circulatory system is most influenced by the intracranial hypertension.

The experiment was carried out on 22 adult mongrel cats. While measuring intracranial pressure continuosly, an extradurally placed rubber balloon was inflated to elevate intracranial pressure. The pressure was elevated to various levels between $300-2,000 \mathrm{mmH}_{2} \mathrm{O}$ and the same pressure was maintained for various length of time between 30 minutes to 12 hours. Rapid serial photographs of the cortical vessels were taken through a glass window tightly placed over the parietal area following to injection of sodium fluorescein $5 \mathrm{mg} / \mathrm{kg} . \mathrm{B}$.W. into inferior vena cava. The series of photographs were taken right before and right after the elevation of the intracranial pressure, and were repeated every 3 hours thereafter. Before the pressure was elevated the dye appears in cortical arteries of approximately $100-300 \mu$ in diameter within 8 seconds after injection of the dye. Within 4 seconds, the dye flows into arterioles and it advances to venule before it disappears from the arterioles. The dye disappears from veins of 
tpproximately $100-300 \mu$ in diameter within 5 seconds following its appearance n the veins. Unless the pressure was elevated to extremely high level ( $900-$ $?, 000 \mathrm{mmH}_{2} \mathrm{O}$ ), the dye appears in the cortical arteries without delay. However, when the pressure reaches to the high level, it delays markedly or sometimes does. not appear at all. When the moderate degree of pressure $\left(400-700 \mathrm{mmH}_{2} \mathrm{O}\right)$ is maintained longer than 6 hours, the dye appears in the venule after it completely disappears from the arterioles. Disappearance of the dye from the veins of above mentioned size is delayed whether the pressure is markedly elevated or after the moderate pressur is maintained certain period of time. No circulatory change is seen when the pressure is below $400 \mathrm{mmH}_{2} \mathrm{O}$. We feel it is important to note that inflow into the cerebral arterial system is not influenced by moderately increased intracranial pressure but it mainly dependent to the pressure difference between the intracranial pressure and systemic blood pressure. We also like to stress that the circulatory disturbance does not become prominent right after the pressure is elevated but it progressively become prominent as the same pressure is maintained, and this circulatory change seems to occur mainly either at capillary level or on the venous side of the cerebral circulation.

Thus, the retardation of intracranial blood flow causes increase in cerebral blood volume and as the result, the intracranial pressure is further elevated.

\title{
g-9. Cerebral Blood Flow and Acute Increased Intracranial Pressue, especially on Luxurious Cerebral Perfusion after Decompression
}

\author{
Tadashi Aiba, Tadao Kano, Norimoto KumagaI, \\ Hideaki NuKII and Junichi KAWAFUCHI \\ Dept. of Neurosurgery, Gumma University
}

\section{g-10. Effect of Increased Intracranial Pressure on Cerebral Hemodynamics}

\author{
Hajime Nagai, Kazuhiro Furuse, Koichi Banno, Atsushi Ikeyama, \\ Shigeru MaEda, Michiaki Hasuo and Shin HoshiKawa \\ 2nd Department of Surgery, Nagoya University School of Medicine
}

In order to investigate the mechanisms whereby the increased intracranial ressure produces arterial hypertension, that is, Cushing phenomenon, studies om the cerebral hemodynamic point of view were carried out on adult mongrel ogs. Serial recordings of respiration, systemic blood pressure, cerebral blood W through the cerebral cortex and brain stem, intracranial pressure and EEG 